\title{
PERSISTENT TOPOLOGY OF SYNTAX
}

\author{
ALEXANDER PORT, IULIA GHEORGHITA, DANIEL GUTH, JOHN M. CLARK, CRYSTAL LIANG, \\ SHIVAL DASU, MATILDE MARCOLLI
}

\begin{abstract}
We study the persistent homology of the data set of syntactic parameters of the world languages. We show that, while homology generators behave erratically over the whole data set, non-trivial persistent homology appears when one restricts to specific language families. Different families exhibit different persistent homology. We focus on the cases of the Indo-European and the Niger-Congo families, for which we compare persistent homology over different cluster filtering values. We investigate the possible significance, in historical linguistic terms, of the presence of persistent generators of the first homology. In particular, we show that the persistent first homology generator we find in the Indo-European family is not due (as one might guess) to the Anglo-Norman bridge in the Indo-European phylogenetic network, but is related to the position of Ancient Greek and the Hellenic branch within the network.
\end{abstract}

\section{INTRODUCTION}

This project is part of a broader ongoing investigation into the use of methods from data analysis to identify the presence of structures and relations between the syntactic parameters of the world languages, considered either globally across all languages, or within specific language families and in comparative analysis between different families.

We analyze the SSWL database of syntactic structures of world languages, using methods from topological data analysis. After performing principal component analysis to reduce the dimensionality of the data set, we compute the persistent homology. The generators behave erratically when computed over the entire set of languages in the database. However, if restricted to specific language families, non-trivial persistent homology appears, which behaves differently for different families. We focus our analysis on the two largest language families covered by the SSWL database: the Niger-Congo family and the Indo-European family. We show that the Indo-European family has a non-trivial persistent generator in the first homology. By performing cluster analysis, we show that the four major language families in the database (Indo-European, Niger-Congo, Austronesian, Afro-Asiatic) exhibit different cluster structures in their syntactic parameters. This allows us to focus on specific cluster filtering values, where other non-trivial persistent homology can be found, in both the Indo-European and the Niger-Congo cases.

This analysis shows that the Indo-European family has a non-trivial persistent generator of the first homology, and two persistent generators of the zeroth homology (persistent connected components), with substructures emerging at specific cluster filtering values. The Niger-Congo family, on the other hand, does not show presence of persistent first homology, and has one persistent connected component.

We discuss the possible linguistic significance of persistent connected components and persistent generators of the first homology. We propose an interpretation of persistent components in terms of subfamilies, and we analyze different possible historical linguistic mechanisms that may give rise to non-trivial persistent first homology.

We focus on the non-trivial persistent first homology generator in the Indo-European family and we try to trace its origin in the structure of the phylogenetic network of Indo-European languages. The first hypothesis we consider is the possibility that the non-trivial loop in the space of syntactic 
parameters may be a reflection of the presence of a non-trivial loop in the phylogenetic network, due to the historical "Anglo-Norman bridge" connecting French to Middle English, hence creating a non-trivial loop between the Latin and the Germanic subtrees. However, we show by analyzing the syntactic parameters of these two subtrees alone that the persistent first homology is not coming from this part of the Indo-European family. We show that it is also not coming from the Indo-Iranian branch. Moreover, we show that adding or removing the Hellenic branch from the remaining group of Indo-European languages causes a change in both the persistent first homology and the number of persistent component.

Acknowledgment. This work was performed within the activities of the last author's Mathematical and Computational Linguistics lab and CS101/Ma191 class at Caltech. The last author is partially supported by NSF grants DMS-1007207, DMS-1201512, and PHY-1205440.

\section{Syntactic parameters And Data Analysis}

The idea of codifying different syntactic structures through parameters is central to the Principles and Parameters model of syntax, [5], 6], within Generative Linguistics. In this approach, one associates to a language a string of binary ( \pm or $0 / 1$ valued) variables, the syntactic parameters, that encode many features of its syntactic structures. Examples of such parameters include Subject $V e r b$, which has the value + when in a clause with intransitive verb the order Subject Verb can be used; Noun Possessor, which has value + when a possessor can follow the noun it modifies; Initial Polar $Q$ Marker, which has value + when a direct yes/no question is marked by a clause initial question-marker; etc. ${ }^{1}$ The "Syntactic Structures of the World's Languages" (SSWL) database, which we used in this investigation, includes a set of 115 different parameters, (partially) mapped for a set of 252 of the known world languages.

The comparative study of syntactic structures across different world languages plays an important role in Linguistics, see [13] for a recent extensive treatment. In particular, in this study, we focus on data of syntactic parameters for two of the major families of world languages: the IndoEuropean family and the Niger-Congo family. These are the two families that are best represented in the SSWL database, which includes 79 Indo-European languages and 49 Niger-Congo languages. The Niger-Congo family is the largest language family in the world (by number of languages it comprises). General studies of syntactic structures of Niger-Congo languages are available, see for instance [2], 12], though many of the languages within this family are still not very well mapped when it comes to their syntactic parameters in the SSWL database. The Indo-European family, on the other hand, is very extensively studied, and more of the syntactic parameters are mapped. Despite this difference, the data available in the SSWL database provide enough material for a comparative data analysis between these two families.

The point of view based on syntactic parameters has also come to play a role in the study of Historical Linguistics and language change, see for instance [8]. An excellent expository account of the parametric approach to syntax is given in [1].

One of the sources of criticism to the Principles and Parameters model is the lack of a good understanding of the space of syntactic parameters, [11. In particular, the theory does not clearly identify a set of independent binary variables that can be thought of as a "universal set of parameters", and relations between syntactic parameters are not sufficiently well understood.

It is only in recent years, however, that accessible online databases of syntactic structures have become available, such as the WALS database of [10] or the SSWL database [14]. The existence of

\footnotetext{
${ }^{1}$ See http://sswl.railsplayground.net/browse/properties for a list and description of all the syntactic parameters covered by the SSWL database.
} 

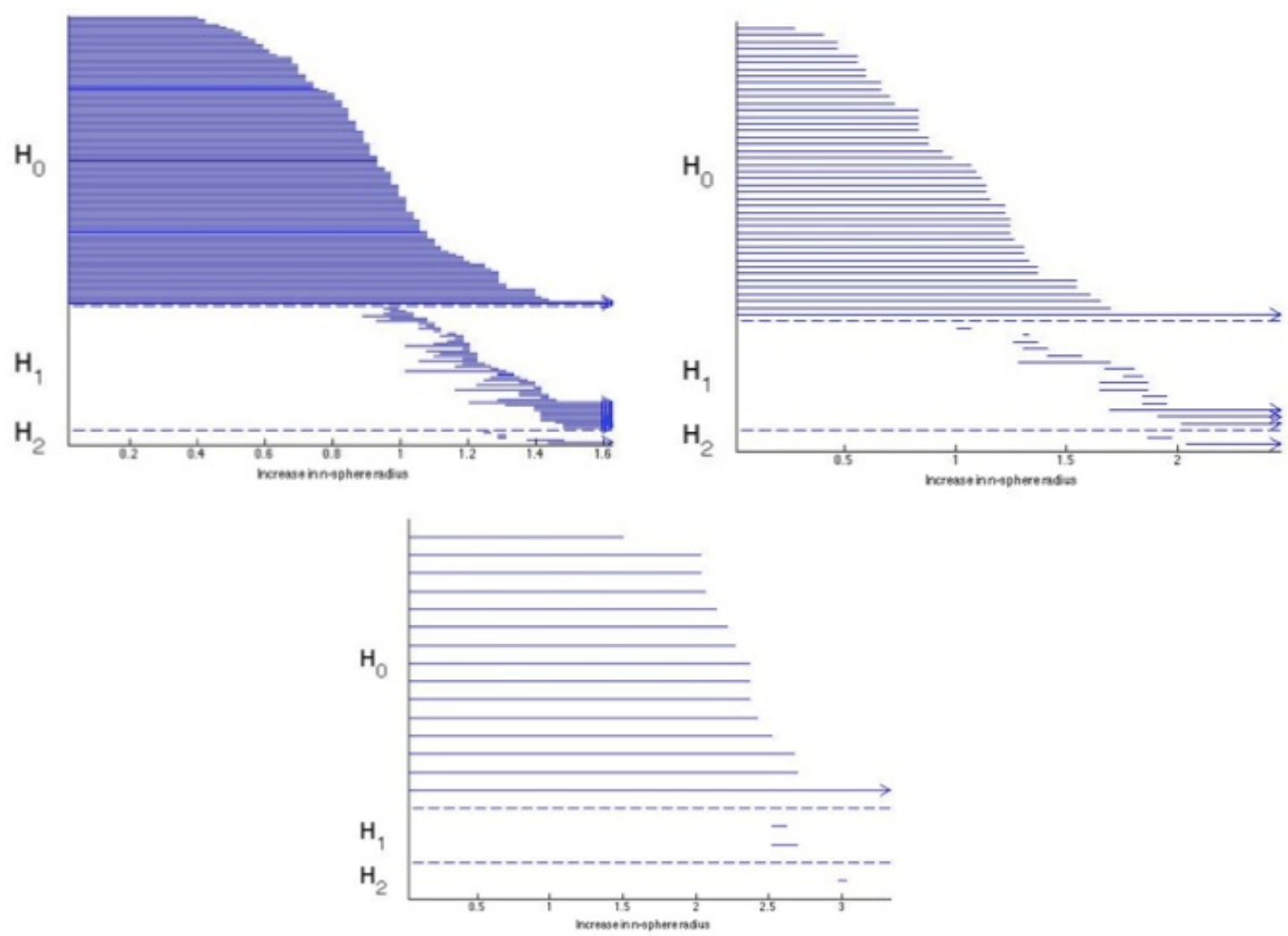

Figure 1. Barcode graph over languages with $60 \%$ of parameters known and $60 \%$ of variance preserved; and with $80 \%$ of parameters known and $60 \%$ of variance preserved. Third graph: barcode for a random subset of 15 languages, $100 \%$ of variance preserved.

databases that record syntactic parameters across different world languages for the first time makes them accessible to techniques of modern data analysis. Our hope is that a computational approach, based on various data analysis techniques applied to the syntactic parameters of world languages, may help the investigation of possible dependence relations between different parameters and a better understanding of their overall structure. In the present study, we focused on the data collected in the SSWL database, and on topological data analysis based on persistent homology.

The structures we observe do not, at present, have a clear explanation in terms of Linguistic theory and of the Principles and Parameters model of syntax. The presence of persistent homology in the syntactic parameter data, and its different behavior for different language families begs for a better understanding of the formation and persistence of topological structures from the Historical Linguistics viewpoint, and from the viewpoint of Syntactic Theory.

\section{Persistent homology}

An important and fast developing area of data analysis, in recent years, has been the study of high dimensional structures in large sets of data points, via topological methods, see [3], [7], [9]. These methods of topological data analysis allow one to infer global features from discrete subsets of data as well as find commonalities of discrete sub-objects from a given continuous object. The techniques developed within this framework have found applications in fields such as pure mathematics (geometric group theory, analysis, coarse geometry), as well as in other sciences (biology, computer science), where one has to deal with large sets of data. Topology is 

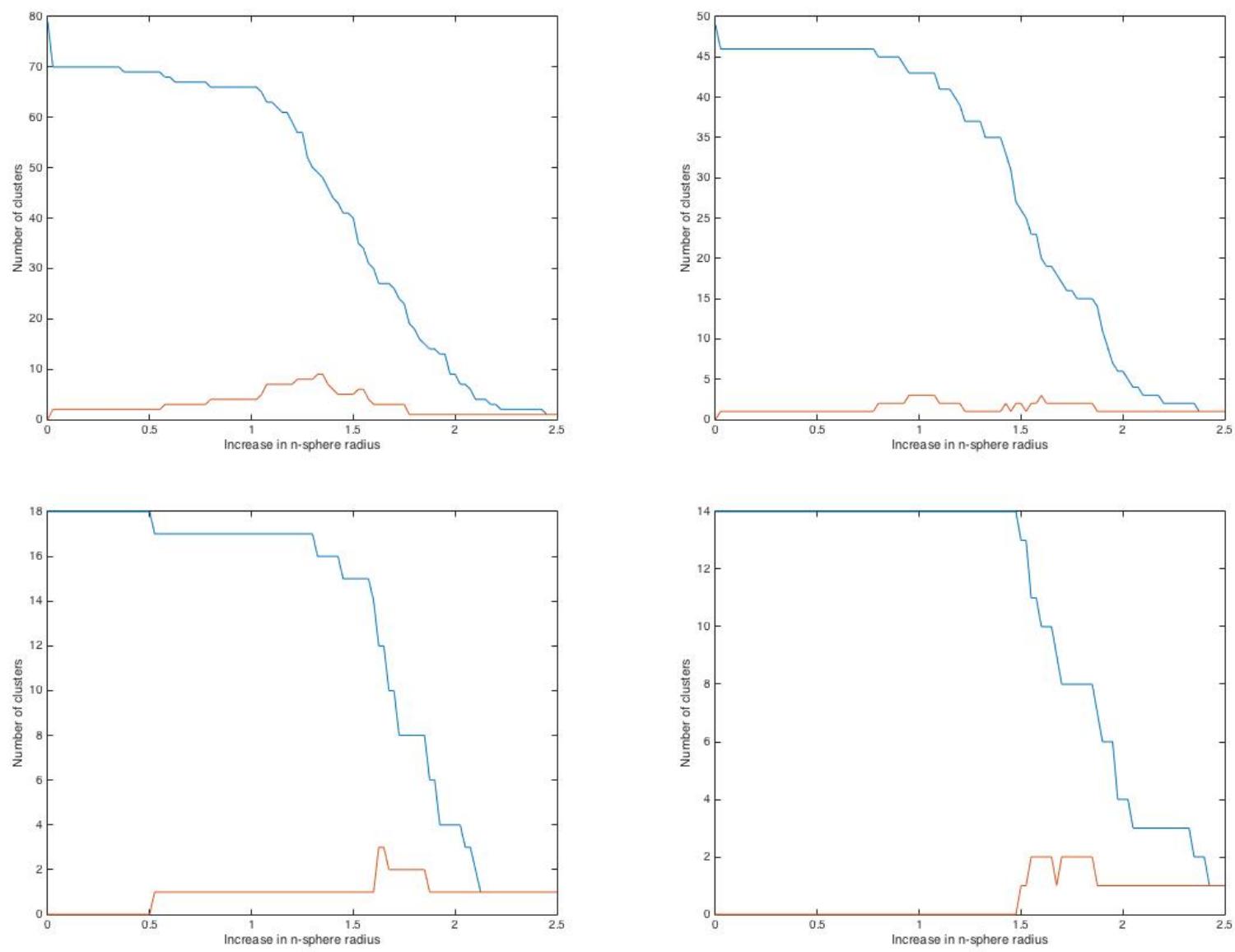

Figure 2. Cluster structure of syntactic parameters for the Indo-European, the Niger-Congo, the Austronesian, and the Afro-Asiatic language families.

very well-suited in tackling these problems, being qualitative in nature. Specifically, topological data analysis achieves its goal by transforming the data set under study into a family of simplicial complexes, indexed by a proximity parameter. One analyzes said complexes by computing their persistent homology, and then encoding the persistent homology of the data set in the form of a parametrized version of a Betti number called a barcode graph. Such graphs exhibit explicitly the number of connected components and of higher-dimensional holes in the data. We refer the reader to [3], [7], [9] for a general overview and a detailed treatment of topological data analysis and persistent homology. As an example, persistent homology was used recently to study the topology of a space of 3D images [4], where the authors determined that the barcode representation from persistent homology matched the homology of a Klein bottle.

3.1. The Vietoris-Rips complex. Suppose given a set $X=\left\{x_{\alpha}\right\}$ of points in some Euclidean space $\mathbb{E}^{N}$. Let $d(x, y)=\|x-y\|=\left(\sum_{j=1}^{N}\left(x_{j}-y_{j}\right)^{2}\right)^{1 / 2}$ denote the Euclidean distance function in $\mathbb{E}^{N}$. The Vietoris-Rips complex $R(X, \epsilon)$ of scale $\epsilon$, over a field $\mathbb{K}$, is defined as the chain complex whose space $R_{n}(X, \epsilon)$ of $n$-simplices corresponds to the $\mathbb{K}$-vector space spanned by all the unordered $(n+1)$-tuples of points $\left\{x_{\alpha_{0}}, x_{\alpha_{1}}, \ldots, x_{\alpha_{n}}\right\}$ where each pair $x_{\alpha_{i}}, x_{\alpha_{j}}$ has distance $d\left(x_{\alpha_{i}}, x_{\alpha_{j}}\right) \leq \epsilon$. The boundary maps $\partial_{n}: R_{n}(X, \epsilon) \rightarrow R_{n-1}(X, \epsilon)$, with $\partial_{n} \circ \partial_{n+1}=0$, are the usual ones determined by the incidence relations of $(n+1)$ and $n$-dimensional simplices. For $n \geq 0$, one 
denotes by

$$
\begin{gathered}
H_{n}(X, \epsilon):=H_{n}(R(X, \epsilon), \partial) \\
=\operatorname{Ker}\left\{\partial_{n}: R_{n}(X, \epsilon) \rightarrow R_{n-1}(X, \epsilon)\right\} / \operatorname{Range}\left\{\partial_{n+1}: R_{n+1}(X, \epsilon) \rightarrow R_{n}(X, \epsilon)\right\}
\end{gathered}
$$

the $n$-th homology with coefficients in $\mathbb{K}$ of the Vietoris-Rips complex. When the scale $\epsilon$ varies, one obtains a system of inclusion maps between the Vietoris-Rips complexes, $R\left(X, \epsilon_{1}\right) \hookrightarrow R\left(X, \epsilon_{2}\right)$, for $\epsilon_{1}<\epsilon_{2}$. By functoriality of homology, these maps induce corresponding morphisms between the homologies, $H_{n}\left(X, \epsilon_{1}\right) \rightarrow H_{n}\left(X, \epsilon_{2}\right)$. A homology class in $H_{n}\left(X, \epsilon_{2}\right)$ that is not in the image of $H_{n}\left(X, \epsilon_{1}\right)$ is a birth; a nontrivial homology class in $H_{n}\left(X, \epsilon_{1}\right)$ that maps to the zero element of $H_{n}\left(X, \epsilon_{2}\right)$ is a death, and a nontrivial homology class in $H_{n}\left(X, \epsilon_{1}\right)$ that maps to a nontrivial homology class in $H_{n}\left(X, \epsilon_{2}\right)$ is said to persist. Mapping the deaths, births, and persistence of a set of generators of the homology, as the radius $\epsilon$ grows gives rise to a barcode graph for the Betti numbers of these homology groups. Those homology generators that survive only over short intervals of $\epsilon$ radii are attributed to noise, while those that persist for longer intervals are considered to represent actual structure in the data set.

3.2. Linguistic significance of persistent homology. When we analyze the persistent topology of different linguistic families (see the detailed discussion of results in \$5), we find different behaviors, in the number of persistent generators in both $H_{0}$ and $H_{1}$. As typically happens in many data sets, the generators for $H_{n}$ with $n \geq 2$ behave too erratically to identify any meaningful structure beyond topological noise.

In general, the rank of the $n$-th homology group $H_{n}$ of a complex counts the "number of $(n+1)$ dimensional holes" that cannot be filled by an $(n+1)$-dimensional patch. In the topological analysis of a point cloud data set, the presence of a non-trivial generator of the $H_{n}$ at a given scale of the Vietoris-Rips complex implies the existence of a set of data points that is well described by an $n$-dimensional set of parameters, whose shape in the ambient space encloses an $(n+1)$ dimensional hole, which is not filled by other data in the same set. In this sense, the presence of generators of persistent homology reveal the presence of structure in the data set.

In our case, the database provides a data point for each recorded world language (or for each language within a given family), and the data points live in the space of syntactic parameters, or in a space of a more manageable lower dimension after performing principal component analysis. In this setting, the presence of an " $(n+1)$-dimensional hole" in the data (a generator of the persistent $H_{n}$ ) shows that (part of) the data cluster around an $n$-dimensional manifold that is not "filled in" by other data points. Possible coordinates on such $n$-dimensional structures represent relations among the syntactic parameters, over certain linguistic (sub)families.

Since the only persistent generators we encountered are in the $H_{0}$ and $H_{1}$, we discuss more in detail their respective meanings.

3.3. Linguistic significance of persistent $H_{0}$. The rank of the persistent $H_{0}$ counts the number of connected components of the Vietoris-Rips complex. It reveals the presence of clusters of data, within which data are closer to each other (in the range of scales considered for the Vietoris-Rips complex) than to any point in any other component. Thus, a language family exhibiting more than one persistent generator of $H_{0}$ has linguistic parameters that naturally group together into different subfamilies. It is not known, at this stage of the analysis, whether in such cases the subsets of languages that belong to the same connected component correspond to historical linguistic subfamilies or whether they cut across them. We will give some evidence, in the case of the IndoEuropean family, in favor of matching persistent generators of the $H_{0}$ to major historical linguistic sub-families within the same family. Certainly, in all cases, the connected components identified 

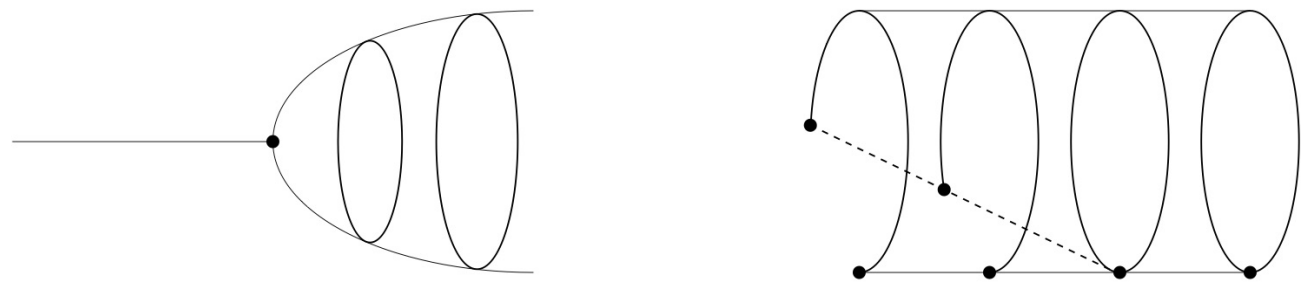

Figure 3. Two models of the development of a non-trivial loop in the space of parameters.

by different generators of the persistent $H_{0}$ can be used to define a grouping into subfamilies, whose relation to historical linguistics remains to be investigated.

3.4. Linguistic significance of persistent $H_{1}$. The presence of an $H_{1}$-generators also means that part of the data (corresponding to one of the components of the Vietoris-Rips complex) clusters around a one-dimensional closed curve. More precisely, one can identify the first homology group $H_{1}(X)$ of a space with the group of homotopy classes $\left[X, S^{1}\right]$ of (basepoint preserving) maps $f: X \rightarrow S^{1}$ to the circle. This means that, if there is a non-trivial generator of the persistent $H_{1}$, then there is a choice of a circle coordinate that best describes that part of the data. The freedom to change the map up to homotopy makes it possible to look for a smoothing of the circle coordinate. It is not obvious how to interpret these circles from the Linguistic point of view. The fact that a generator of the $H_{1}$ represents a 2-dimensional hole means that, given the data that cluster along this circle, no further data point determine a 2-dimensional surface interpolating across the circle. As the topological structures we are investigating stem from a Vietoris-Rips complex that measures proximity between syntactic parameters of different languages, we can propose a heuristic interpretation for the presence of such circles as the case of a (sub)family of languages where each language in the subfamily has other "neighboring" languages with sufficiently similar syntactic parameters, so that one can go around the whole subfamily via changes of syntactic parameters described by a single circle coordinate, while parameter changes that move along two-dimensional manifolds and interpolate between data points on the circle cannot be performed while remain within the same (sub)family.

Two different possible models of how a non-trivial generator of the persistent first homology can arise point to different possible explanations in historical-linguistic terms. As shown in Figure 3. the first model is a typical Hopf bifurcation picture, where a circle arises from a point (with the horizontal direction as time coordinate). This model would be compatible with a phylogenetic network of the corresponding language family that is a tree, where one of the nodes generates a set of daughter nodes whose points in the parameter space contain a nontrivial loop. The second possibility is of a line closing up into a circle. This may arise in the case of a language family whose phylogenetic network is not a tree, but it contains itself a loop that closes off two previously distant branches. There are well known cases where the phylogenetic network of a language family is not necessarily best described by a tree. The most famous case is probably the Anglo-Norman bridge in the phylogenetic "tree" of the Indo-European languages, see Figure 4. However, it is important to point out that the presence of a loop in the phylogenetic network of a language family does not imply that this loop will leave a trace in the syntactic parameters, in the form of a non-trivial first persistent homology. Conversely, the presence of persistent first homology, by itself, is no guarantee that loops may be present in the phylogenetic network, for example due to possibilities like the Hopf bifurcation picture described above. Thus, one cannot infer directly from the presence or absence of a persistent $H_{1}$ conclusions about the topology of the historical 


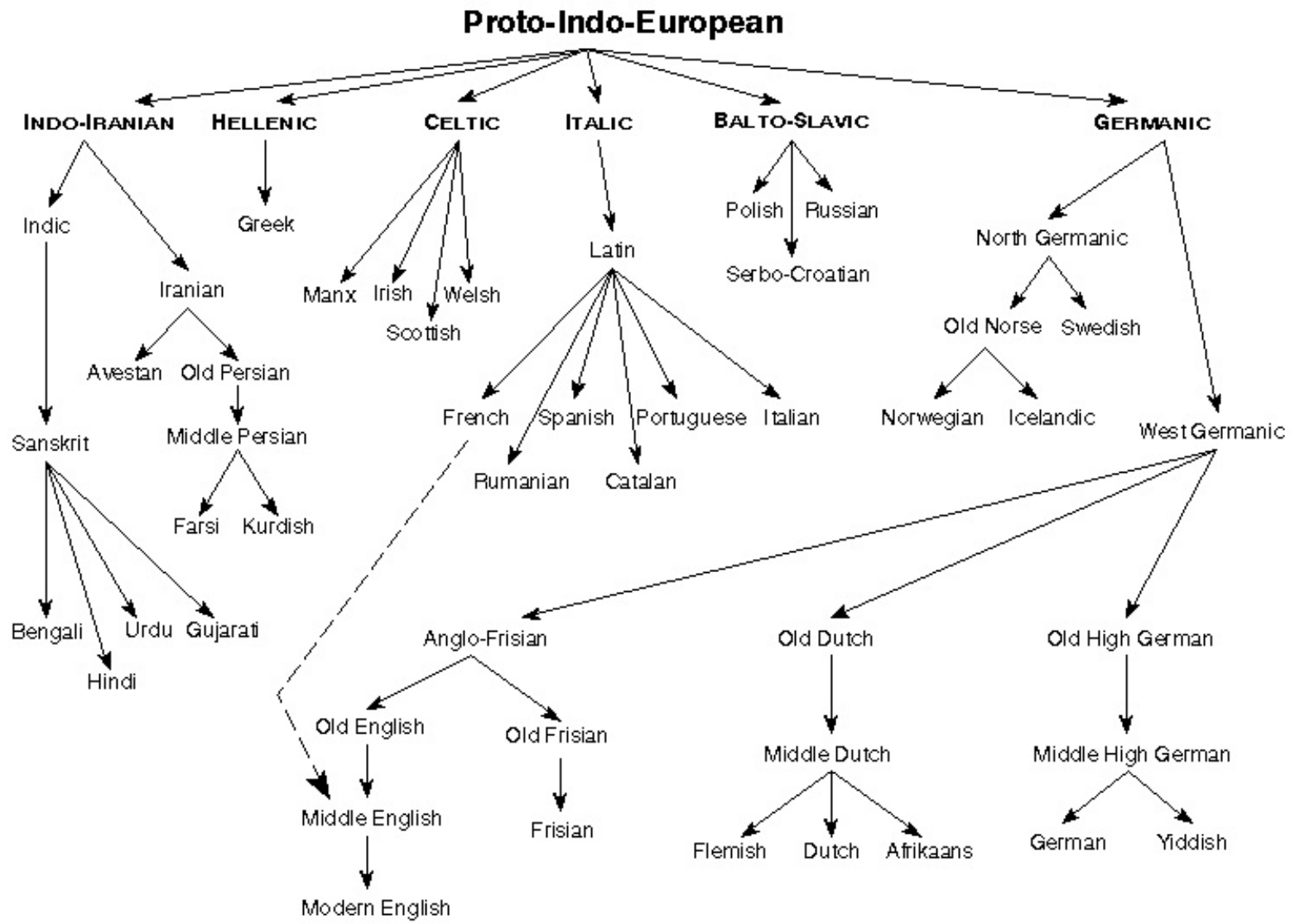

Frepared by Jack Lynch, jlyndh@andromeda. nugers. edx

Figure 4. The family "tree" of the Indo-European languages (by Jack Lynch) showing the loop between the Latin and the Germanic subtrees.

phylogenetic network. The only conclusion of this sort that can be drawn is that a persistent $H_{1}$ suggests a phylogenetic loop as one of the possible causes. Conversely, one can read the absence of non-trivial persistent first homology as a suggestion (but not an implication) of the fact that the phylogenetic network may be a tree and that phenomena like the Anglo-Norman bridge did not occur in the historical development of that family.

We will discuss this point more in detail in the case of the Indo-European language family. This is a very good example, which shows how the possible correlation between loops in the space of syntactic parameters and in the phylogenetic network is by no means an implication. Indeed, the Indo-European language family contains both a known loop in the phylogenetic network, due to the Anglo-Norman bridge (see Figure 4), and a non-trivial generator of the persistent $H_{1}$. However, we will show using our topological data analysis method that these two loops are in fact unrelated, contrary to what intuition might have suggested.

\section{Data Analysis Procedure}

The SSWL database [14] was first imported into a pivot table in Excel. The on-off parameters are represented in binary, in order to compute the distances between languages. However, the parameter values are not known for many of the languages in the database: over one hundred of the languages have, at present, less than half of their parameters known. Thus, we decided to replace empty language parameters with a value of 0.5. All together, we ended up with 252 languages, each with 115 different parameters. 

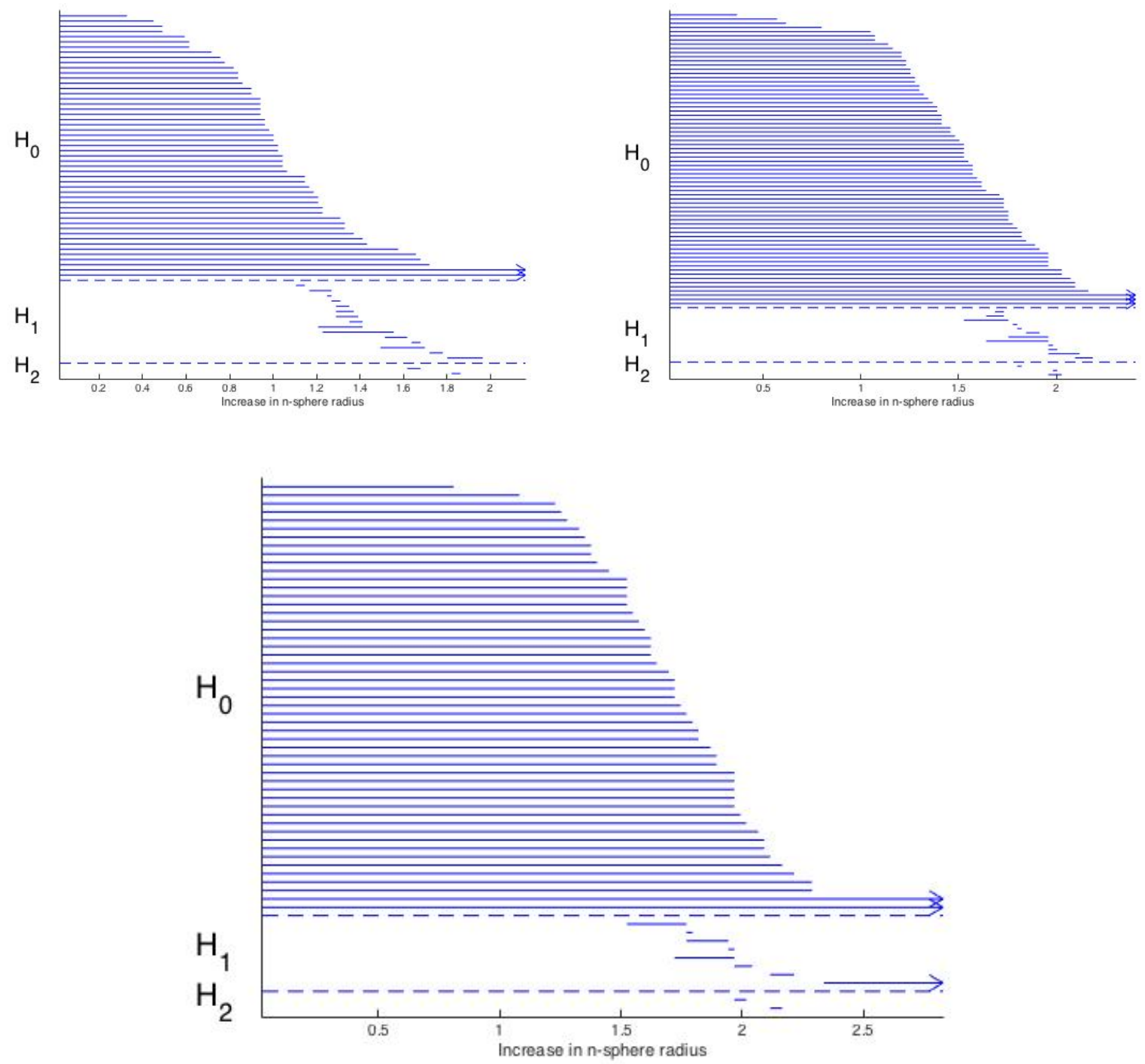

Figure 5. Barcode diagram for the Indo-European language family, at indices $(7,5,96),(10,0,96)$, and $(10,5,98)$.

We then proceeded to our analysis based on the results from Perseus homology software [15]. This is achieved through a series of Matlab scripts? The script named data_select_full.m allows for selection of subsets of the raw data. It performs Principal Component Analysis on the raw parameter data and saves it to a text file for use in Perseus. The format of the data is that of a Vietoris-Rips complex. This script has two important parameters: a completeness threshold, and a percent variance to preserve. The completeness threshold removes the languages below a threshold of known parameters. The percent variance allows us to reduce the dimensionality of our data.

The next script, named barcode.m, was used to create barcode graphs for data visualization. Perseus outputs the birth and death times for each persistent homology generator, which are then used to construct the barcode graph of the persistence intervals to visualize the structure and

\footnotetext{
${ }^{2} \mathrm{~A}$ repository of the code used for this project is available at https://github.com/cosmicomic/cs101-project5
} 

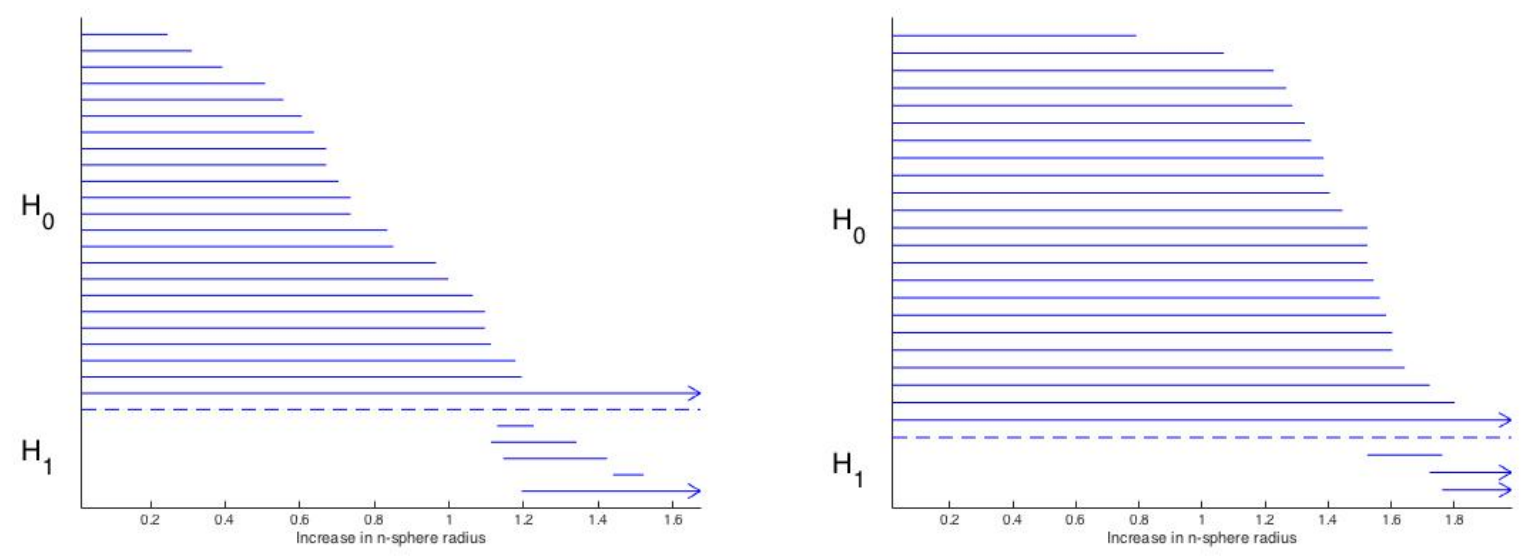

FiguRE 6. Barcode diagram for the Indo-European language family, for cluster filtering value 165 at indices $(7,5,95)$ and $(10,5,95)$.

determine the generators. The radii in our complexes are incremented by $1 \%$ of the mean distance between languages.

Data analysis was initially set up as a three step process: select the data with the script data_select_full.m, analyze it with Perseus, and use barcode.m to visualize the results. The final script, named run_all, streamlines this process under a single input command.

Finally, our analysis includes examining how many data points belong to clusters of points at any given radius. Clusters are constructed by creating $n$-spheres of uniform radius centered at each data point. If the $n$-spheres of two data points overlap, then those data points are in the same cluster. A non-trivial cluster is a cluster with at least two data points contained within. The scripts group_select.m and graph_clusters.m make it possible to visualize the number of clusters and non-trivial clusters as radius increases.

\section{THE PERSISTENT TOPOLOGY OF LINGUISTIC FAMILIES}

A preliminary analysis performed over the entire set of languages in the SSWL database shows that the non-trivial homology generators of $H_{1}$ and $H_{2}$ behave erratically. Moreover, there are too many generators of $H_{2}$ and $H_{3}$ to draw any meaningful conclusion about the structure of the underlying topological space. One can see the typical behavior represented in Figure 1. In the first graph of Figure 1 we included the languages with more than $60 \%$ of the parameters known, while in the second we removed all languages with more than $20 \%$ of the parameters unaccounted for. Here percentage of parameters is with respect to the largest number of syntactic parameters considered in the SSWL database. One can compare this with the case of a randomly generated subset of languages, presented in the third graph of Figure 1. Notice that, while in the cases represented in the first two graphs of Figure 1 there is "noise" in the $H_{1}$ and $H_{2}$ region, that prevents a clear identification of persistent generators, the homology of random subsets of the data, as displayed in the third graph of Figure 1, is relatively sparse, containing only topologically trivial information. This observation lead us to the hypothesis that the behavior seen in Figure 1 stems from a superposition of some more precise, but non-uniform, topological information associated to the various different linguistic families. In order to test this hypothesis, we decided to examine specific language families as an additional method of data filtering. We chose the four largest families represented in the original database: Indo-European with 79 languages, NigerCongo with 49, Austronesian with 18, and Afro-Asiatic with 14. Although some of the languages 

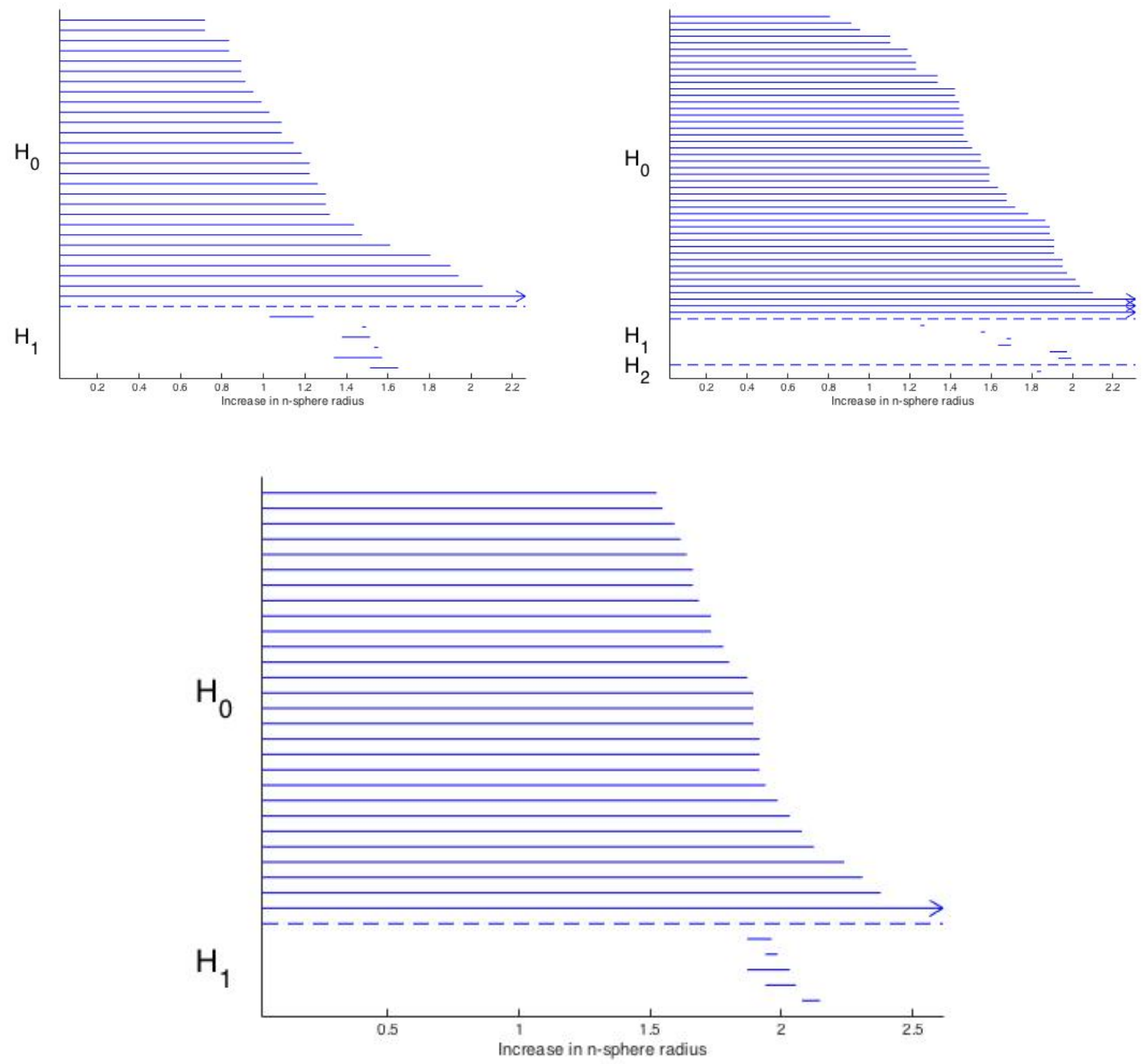

Figure 7. Barcode diagram for the Niger-Congo language family, at indices $(7,5,107),(10,0,100)$, and $(10,5,105)$.

in the database included latitude and longitude coordinates, these were ignored when determining language family.

5.1. Cluster structures in major language families. A first observation, when comparing syntactic parameters of different linguistic families, is that they exhibit different cluster structure of the syntactic parameters. This is illustrated in Figure 2, in the case of the our largest families in the SSWL database.

Based on this cluster analysis, we then focused on the cases of the Indo-European and the Niger-Congo language family and we searched for nontrivial generators of the first homology $H_{1}$ in appropriate ranges of cluster filtering.

The cluster analysis of Figure 2 suggests that cluster filter values between 150 and 200 should provide interesting information. We computed additional barcode diagrams corresponding to cluster filtering values 165 and 190 . 

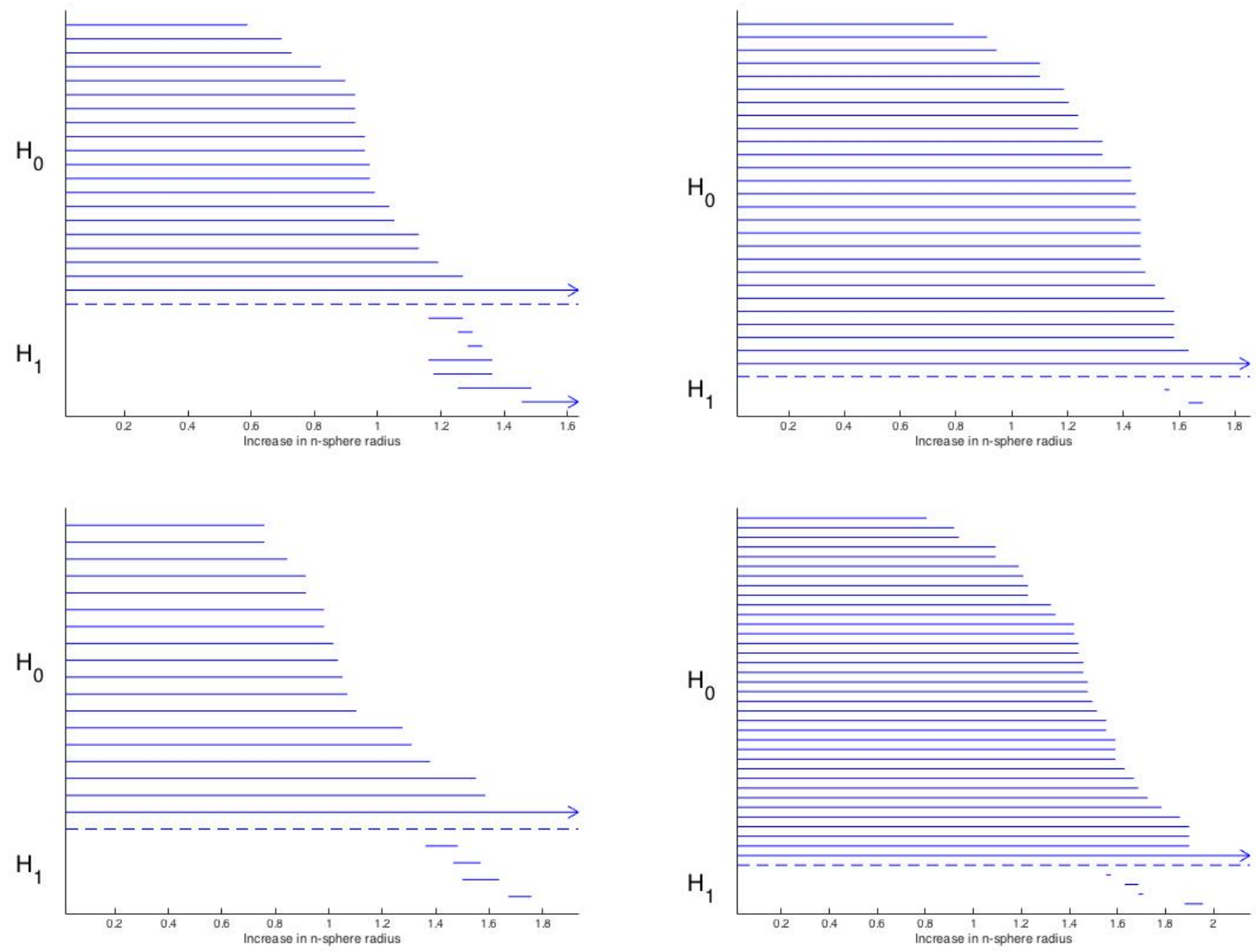

Figure 8. Barcode diagram for the Niger-Congo language family, for cluster filtering value 165 and indices $(7,3,100)$ and $(10,0,100)$, and for cluster filtering value 190 and indices $(7,5,104)$ and $(10,0,104)$.

5.2. Indexing in barcode graphs. In the graphs presented in the following subsection, the barcode graphs are labeled by a set of three indices. The first two indices refer to the Principal Component Analysis and the third index to the runs of the Perseus program computing births and deaths of homology generators of the Vietoris-Rips complex. More precisely, the first index ( 7 or 10) refers to the percent variance divided by 10 , while the second index $(0,3$ or 5$)$ refers to the percent complete divided by 10. They are discussed above in $\S 4$. The third parameter is the number of steps in Perseus. If present, the additional parameter given by the number after "cluster" is one hundred times the radius used for cluster filtering.

5.3. Persistent topology of the Indo-European family. We analyzed the persistent homology of the syntactic parameters for the Indo-European language family. As shown in Figure 5 , at values $(7,5,96)$ and $(10,0,96)$ one sees persistent generators of $H_{0}$ and intervals in the varying $n$-sphere radius, for which nontrivial $H_{1}$ generators exist. At values $(10,5,98)$, as shown in Figure 5. one sees one persistent generator of $H_{1}$ and two persistent generators of $H_{0}$. The existence of a persistent generator for the $H_{1}$ suggests that there should be a "circle coordinate" description for at part of the syntactic parameters of the Indo-European languages. The fact that there are two persistent generators of $H_{0}$ in the same diagram indicates two connected components, only one of which is a circle: this component determines which subset of syntactic parameters admits a parameterization by values of a circle coordinate. 

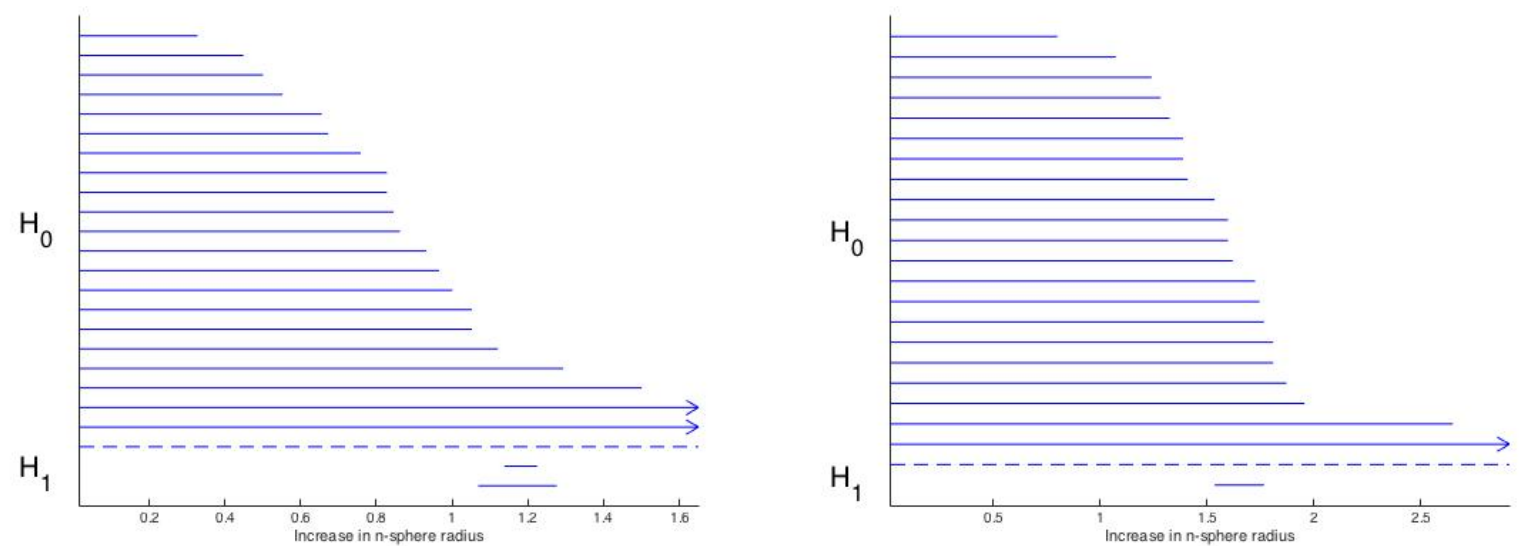

Figure 9. Barcode diagram for the Latin+Germanic languages, at indices $(7,5,129)$ and $(10,5,130)$.

Based on the cluster analysis described in \$5.1 above, we then focused on specific regions of cluster filtering values that were more likely to exhibit interesting topology. For example, for cluster filtering value 165, the results show, respectively, one generator of $H_{0}$ and one generator of $H_{1}$, for indices $(7,5,95)$, and one generator of $H_{0}$ and a possibility of two persistent generators of the $H_{1}$, for indices $(10,5,95)$, see Figure 6. The appearance of persistent generators of the $H_{1}$ as specific cluster filtering values identifies other groups of syntactic parameters that may admit circle variable parameterizations. What these topological structures in the space of syntactic parameters, and these subsets admitting circle variables description, mean in terms of linguistic theory remains to be fully understood. We analyze some historical-linguistic hypotheses in the following subsection.

5.4. Indo-European persistent topology and historical linguistics. It is often argued that the phylogenetic "tree" of the family of Indo-European languages should not really be a tree, because of the historical influence of French on Middle English, see Figure 6, which can be viewed as creating a bridge (sometimes referred to as the Anglo-Norman bridge) connecting the Latin and the Germanic subtrees and introducing non-trivial topology in the Indo-European phylogenetic network. It is well known that the influx of French was extensive at the lexical level, but it is not clear whether one should expect to see a trace of this historical phenomenon when analyzing languages at the level of syntactic structures. It is, however, a natural question to ask whether the non-trivial loop one sees in the persistent topology of syntactic parameters of the Indo-European family may perhaps be a syntactic remnant of the Anglo-Norman bridge.

However, a further analysis of the SSWL dataset of syntactic parameters appears to exclude this possibility. Indeed, we computed the persistent homology using only the Indo-European languages in the Latin and Germanic groups. If the persistent generator of $H_{1}$ were due to the Anglo-Norman bridge one would still find this non-trivial generator when using only this group of languages, while what we find is that the group of Latin and Germanic languages alone carry no non-trivial persistent first homology, see Figure 9.

In order to understand the nature of the two persistent generators of $H_{0}$, we separated out the Indo-Iranian subfamily of the Indo-European family, to test whether the two persistent connected components would be related to the natural subdivision into the two main branches of the IndoEuropean family. Even though the Indo-Iranian branch is the largest subfamily of Indo-European languages, it is much less extensively mapped in SSWL than the rest of the Indo-European family, 


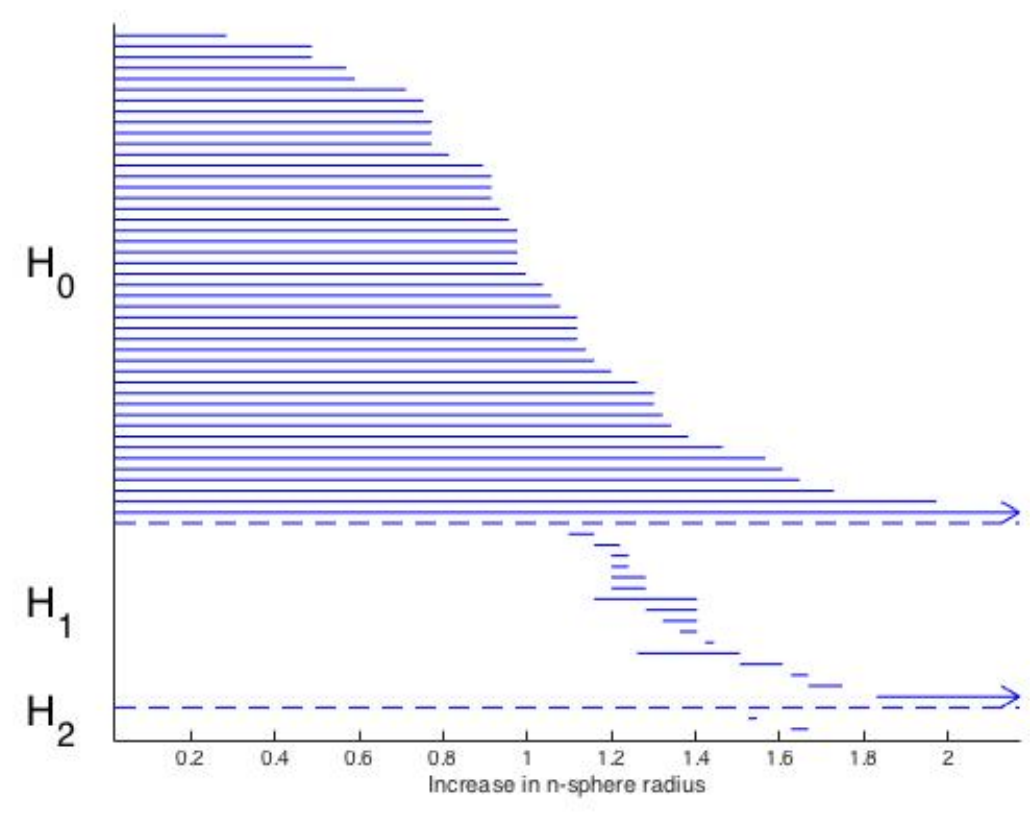

FiguRE 10. Barcode diagram for the Indo-European family with the Indo-Iranian subfamily removed, at indices $(7,5,97)$.

with only 9 languages recorded in the database. Thus, a topological data analysis performed directly on the Indo-Iranian subfamily is less reliable, but one can gain sufficient information by analyzing the remaining set of Indo-European languages, after removing the Indo-Iranian subfamily. The result is illustrated in Figure 10. We see that indeed the number of persistent connected component is now just one, which supports the proposal of relating persistent generators of $H_{0}$ to major subdivisions into historical linguistic subfamilies. Moreover, the persistent generator of the $H_{1}$ is still present, which shows that the non-trivial first homology is not located in the Indo-Iranian subfamily.

In order to understand more precisely where the non-trivial persistent first homology is located in the Indo-European family, we performed the analysis again, after removing the Indo-Iranian languages and also removing the Hellenic branch, including both Ancient and Modern Greek. The resulting persistent topology is illustrated in Figure 11. By comparing Figures 10 and 11 one sees that the position of the Hellenic branch of the Indo-European family has a direct role in determining the persistent topology. When this subfamily is removed, the number of persistent connected components (generators of $H_{0}$ ) jumps from one to three, while the non-trivial single generator of $H_{1}$ disappears. Although this observation by itself does not provide an explanation of the persistent topology in terms of historical linguistics of the Indo-European family, it points to the fact that, if historical linguistic phenomena are involved in determining the topology, they appear to be related to the role that Ancient Greek and the Hellenic branch played in the historical development of the Indo-European languages.

When performing a more detailed cluster analysis on the Indo-European family, one finds substructures in the persistent topology. For instance, as shown in Figure 6, one sees a possible second generator of the persistent $H_{1}$ for cluster filtering value 165 , with indices $(10,5,95)$. These substructures may also be possible traces of other historical linguistic phenomena. 


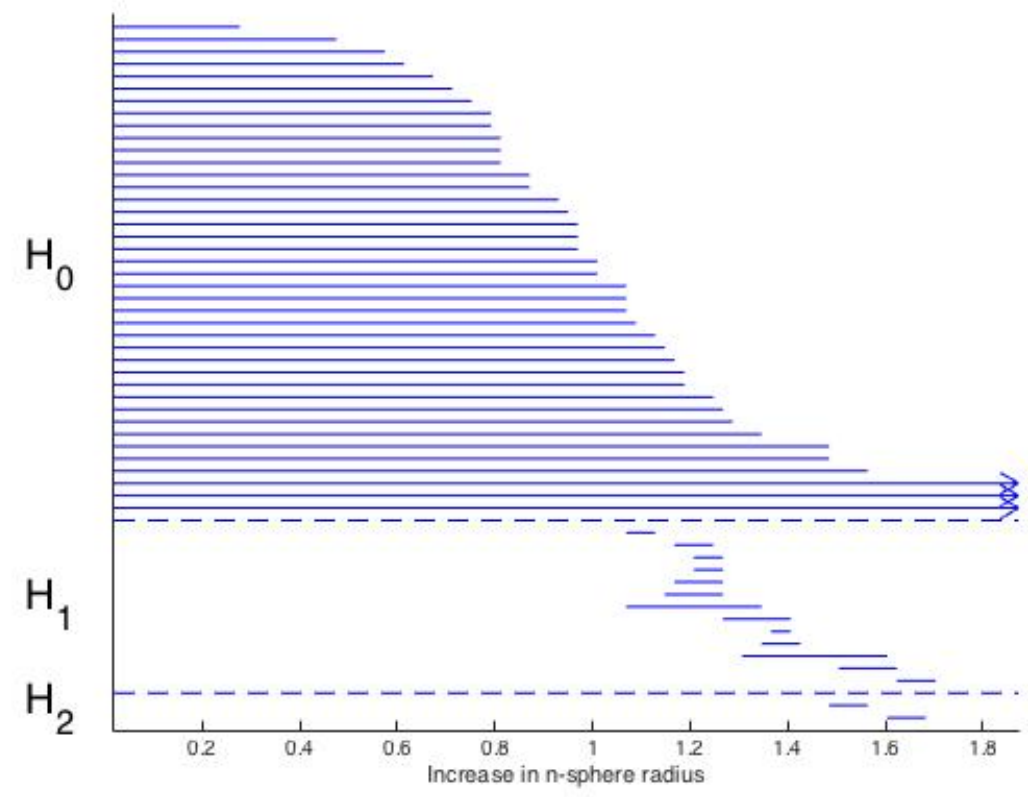

FiguRE 11. Barcode diagram for the Indo-European family with the Indo-Iranian and the Hellenic subfamilies removed, at indices $(7,5,96)$.

5.5. Persistent topology of the Niger-Congo family. We performed the same type of analysis on the syntactic parameters of the Niger-Congo language family. The interesting result we observed is that the behavior of persistent homology seems to be quite different for different language families. Figure 7 shows the barcode diagrams for persistent homology at index values $(7,5,107)$, $(10,0,100)$, and $(10,5,105)$, which can be compared with the diagrams of Figure 5 for the IndoEuropean family. In the Niger-Congo family, we now see persistent $H_{0}$ homology, respectively, of ranks 1, 3, and 1 (compare with ranks 2, 3, 2 in the Indo-European case). A lower rank in the $H_{0}$ means fewer connected components in the Vietoris-Rips complex, which seems to indicate that the syntactic parameters are more concentrated and homogeneously distributed across the linguistic family, and less "spread out" into different sub-clusters.

Following the cluster analysis of $\$ 5.1$, we also considered the persistent homology for the NigerCongo family at specific cluster filtering values. While for cluster filtering value 165 and indices $(7,3,100)$ one sees one persistent generator of $H_{0}$ and a possibility of a persistent generator in the $H_{1}$, cluster filtering value 165 with indices $(10,0,100)$, as well as cluster filtering value 190 with indices $(7,5,104)$ and $(10,0,104)$ only show one persistent generator in the $H_{0}$.

This persistent homology viewpoint seems to suggest that syntactic parameters within the Niger-Congo language family may be spread out more evenly across the family than they are in the Indo-European case, with a single persistent connected component, whereas the Indo-European ones have two different persistent connected component, one of which has circle topology.

\section{FURTHER QUESTIONS}

We showed that methods from topological data analysis, in particular persistent homology, can be used to analyze how syntactic parameters are distributed over different language families. In particular we compared the cases of Indo-European and Niger-Congo languages. 
We list here some questions that naturally arise from this perspective, which we believe are worthy of further investigation.

(1) To what extent do persistent generators of the $H_{0}$ (that is, the persistent connected components) of the data space of syntactic parameter correspond to different (sub)families of languages in the historical linguistic sense? For example, are the three $H_{0}$ generators visible at scale $(10,0,100)$ in the Congo-Niger family a remnant of the historical subdivision into the Mande, Atlantic-Congo, and Kordofanian subfamilies?

(2) What is the meaning, in historical linguistic terms, of the circle components (persistent generators of $H_{1}$ ) in the data space of syntactic parameters of language families? Is there a historical-linguistic interpretation for the second $H_{1}$ generator one sees at cluster filtering value 165 and scale $(10,5,95)$ in the Indo-European family? Or for the $H_{1}$ generator one sees with the same cluster filtering, at scale $(7,3,100)$ in the Niger-Congo case?

(3) To what extent does persistent topology describe different distribution of syntactic parameters across languages for different linguistic families?

\section{REFERENCES}

[1] M. Baker, The Atoms of Language, Basic Books, 2001.

[2] J. Bendor-Samuel, The Niger-Congo languages: a classification and description of Africa's largest language family, University Press of America, 1989.

[3] G. Carlsson, Topology and Data, Bulletin of the American Mathematical Society, Vol. 46 (2009) N.2, 255-308.

[4] G. Carlsson, T. Ishkhanov, V. de Silva, A. Zomorodian, On the local behavior of spaces of natural images, Int. J. Comput. Vis., Vol.76 (2008) 1-12.

[5] N. Chomsky, Lectures on Government and Binding, Dordrecht: Foris Publications, 1982.

[6] N. Chomsky, H. Lasnik, The theory of Principles and Parameters, in "Syntax: An international handbook of contemporary research", pp.506-569, de Gruyter, 1993.

[7] H. Edelsbrunner, J.L. Harer, Computational Topology: An Introduction, American Mathematical Society, 2010.

[8] C. Galves (Ed.) Parameter Theory and Linguistic Change, Oxford University Press, 2012.

[9] R. Ghrist, Elementary Applied Topology, CreateSpace, 2014.

[10] M. Haspelmath, M.S. Dryer, D. Gil, B. Comrie, The World Atlas of Language Structures, Oxford University Press, 2005.

[11] M. Haspelmath, Parametric versus functional explanations of syntactic universals, in "The limits of syntactic variation", pp. 75-107, John Benjamins, 2008.

[12] V. Manfredi, K. Reynolds, (Eds.) Niger-Congo Syntax and Semantics, Boston University, African Studies Center, 1995.

[13] T. Shopen, Language Typology and Syntactic Description: Volume 1, Clause Structure; Volume 2, Complex Constructions; Volume 3: Grammatical Categories and Lexicon, Cambridge University Press, 2007.

[14] SSWL Database of Syntactic Parameters: http://sswl.railsplayground.net/

[15] Perseus software package for persistent homology http://wWw.sas.upenn.edu/ vnanda/perseus/

Division of Physics, Mathematics and Astronomy, Caltech, 1200 E. California Blvd. Pasadena, CA 91125, USA

E-mail address: aport@caltech.edu

E-mail address: igheorgh@caltech.edu

E-mail address: dguth@caltech.edu

E-mail address: jmclark@caltech.edu

E-mail address: clliang@caltech.edu

E-mail address: sdasu@caltech.edu

E-mail address: matilde@caltech.edu 